\title{
Propriedades psicométricas da Escala de Atitudes Alimentares Transtornadas para adultos do sexo masculino
}

\author{
Psychometric properties of the Disordered \\ Eating Attitude Scale for adult men
}

Marle dos Santos Alvarenga', Pedro H. B. Carvalho², Sonia T. Philippi', Fernanda B. Scagliusi

\section{RESUMO}

Objetivo: Avaliar as propriedades psicométricas da Escala de Atitudes Alimentares Transtornadas (EAAT) para o sexo masculino. Métodos: Duzentos e vinte e oito universitários (18-39 anos) responderam à EAAT, originalmente desenvolvida e validada para o sexo feminino. A consistência interna foi avaliada pelo Alpha de Cronbach e a validade convergente, por meio do coeficiente de correlação de Pearson comparando os escores da EAAT, do Teste de Atitudes Alimentares (EAT) e da Escala de Restrição (RS). A reprodutibilidade foi avaliada aplicando a escala numa subamostra $(n=38)$ com um mês de intervalo utilizando o coeficiente de correlação intraclasse (CCl). A validade known-groups foi obtida comparando o escore dos universitários na EAAT com o escore de homens com diagnóstico de transtornos alimentares (TA) $(n=28)$. Resultados: A consistência interna da escala foi de 0,63. O escore da EAAT foi correlacionado com a EAT $(r=0,65)$ e RS $(r=0,51)$, e o CCl entre o teste e o reteste foi de 0,948. A análise known-groups diferenciou pacientes com TA de estudantes universitários ( $p<$

\section{Palavras-chave}

Homens, atitude alimentar, psicometria, validade, transtornos alimentares.
0,001). Conclusões: A escala apresentou propriedades psicométricas adequadas e pode ser utilizada em estudos com homens adultos - uma vez que o constructo é pouco explorado em homens. Recomenda-se, de qualquer forma, uma revisão da escala e desenvolvimento de instrumentos específicos para o público masculino.

\begin{abstract}
Objective: To evaluate psychometric properties of the Disordered Eating Attitude Scale (DEAS) for men. Methods: Two hundred and twenty-eight undergraduate male students (18-39 years old) answered the DEAS, originally developed and validated for women. Internal consistency was evaluated by Cronbach's Alpha; convergent validity by comparing DEAS and the Eating Attitude (EAT) and Restraint Scale (RS) scores using Pearson's coefficient. Test-retest reliability was evaluated with a subsample $(n=38)$ in a month interval by means of intraclass correlation coefficient (ICC). Known-groups validity was obtained comparing scores in DEAS among undergraduate students and men with eating disorders (ED) $(n=28)$. Results: Internal consistency of scale was 0.63. DEAS score correlated with EAT $(r=0.65)$ and RS $(r=$
\end{abstract}

1 Universidade de São Paulo, Faculdade de Saúde Pública (USP/FSP), Departamento de Nutrição.

2 Universidade Federal de Juiz de Fora (UFJF), Programa de Pós-Graduação em Psicologia. 


\section{Keywords}

Men, eating attitude, psychometrics, validity, eating disorders.
0.51); ICC between test and retest was 0.948. Known-groups analysis differentiated ED patients and undergraduate students $(p<0.001)$. Conclusions: The scale presented adequate psychometric properties and could be used in studies with adult men, since the construct is not explored among males. Nevertheless, it is recommended to revise the scale and to develop specific instruments for male public.

\section{INTRODUÇÃO}

Atitudes alimentares englobam crenças, pensamentos, sentimentos, comportamentos e relacionamento para com os alimentos ${ }^{1}$ e podem ser boas preditoras da ingestão alimentar $^{2}$, ou seja, quem tem uma relação mais positiva com o alimento pode fazer melhores escolhas alimentares.

Atitudes alimentares disfuncionais envolvem conceitos rígidos sobre alimentação saudável, culpa, medo e ansiedade relacionados às escolhas alimentares e ao comer, pensar obsessivamente sobre alimentos e calorias, usar a comida para compensar problemas emocionais, entre outros ${ }^{3}$.

Por se saber que problemas alimentares são mais comuns em mulheres, a maior parte dos estudos foca apenas o grupo feminino ${ }^{4,5}$. Mas o público masculino também deve ser alvo de atenção sobre possíveis atitudes alimentares disfuncionais ${ }^{6-8}$. As demandas complexas sobre "alimentação mais saudável" e a preocupação com a imagem corporal podem impactar as escolhas e atitudes alimentares.

$O$ aumento da incidência de transtornos alimentares (TA) em homens chama atenção para a necessidade de maior compreensão da relação para com o alimento no sexo masculino ${ }^{6-9}$. Ademais, acredita-se que existam subnotificações em relação aos TA em homens, bem como pode haver atualmente um aumento do alcance de trabalho de pesquisa sobre essa temática, indicando que os TA em homens podem se apresentar de forma diferenciada ${ }^{79}$. A busca pela muscularidade, preocupação central dos homens, é um dos fatores de risco para o desenvolvimento de transtornos mentais, como é o caso da dismorfia muscular ${ }^{6,9}$. Dessa forma, é de interesse avaliar como a população masculina tem lidado com as questões corporais e, por consequência, com as questões alimentares.

Alguns instrumentos disponíveis avaliam atitudes alimentares específicas, como a Restraint $S_{c a l e}{ }^{10}$ e a Eating Attitudes Test ${ }^{11}$; muitos desses têm um foco exclusivo em pacientes com TA - avaliando sintomas dos quadros; e outros têm como foco a seleção de alimentos e não exatamente a relação do indivíduo para com a alimentação'.

A Escala de Atitudes Alimentares Transtornadas (EAAT) foi desenvolvida e validada no Brasil para população adulta feminina (estudantes universitárias), definindo o constructo "atitude alimentar" como crenças, pensamentos, sentimentos, comportamentos e relacionamento para com os alimentos ${ }^{1}$. A escala foi também validada para o mesmo público em inglês, espanhol e japonês ${ }^{12-14}$, mantendo boas propriedades psicométricas.

As evidências de validade da EAAT foram também investigadas entre adolescentes de ambos os sexos ${ }^{15}$, mostrando algumas diferenças entre meninos e meninas. Para ampliar as possibilidades de aplicação da escala, suas propriedades psicométricas devem ser também avaliadas para adultos do sexo masculino.

A EAAT pode ser útil no estudo de diferentes populações, esclarecendo diferenças entre grupos, países, culturas, grupos étnicos - e entre populações clínicas. Nesse contexto, o objetivo deste estudo foi avaliar a validade da Escala de Atitudes Alimentares Transtornadas para o sexo masculino.

\section{MÉTODOS}

\section{Escala de Atitudes Alimentares Transtornadas}

A escala desenvolvida para avaliação de mulheres jovens foi utilizada da mesma maneira na avaliação do sexo masculino - foram feitas apenas mudanças de artigo "A" para "O" nas questões nas quais isso era pertinente. Um estudo preliminar utilizou a escala dessa maneira com homens com diagnóstico de transtornos alimentares, sem encontrar problemas de compreensão8.

A EAAT possui 25 questões, pontuadas em escala na forma Likert de pontos, sendo que maiores pontuações indicam atitudes mais disfuncionais; a pontuação mínima possível é de 37 pontos e a máxima, de 190 pontos. A escala possui cinco subescalas: Fator 1 - Relação para com o alimento; Fator 2 - Preocupação com alimentação e ganho de peso; Fator 3 - Práticas restritivas e compensatórias; Fator 4 - Sentimentos em relação à alimentação; e Fator 5 - Conceito de alimentação normal'.

A avaliação psicométrica da escala em seu desenvolvimento original demonstrou que ela possui adequada consistência interna (Alpha de Cronbach $=0,7$ ); boa validade convergente - avaliada na correlação da pontuação com o Teste de Atitudes Alimentares $(r=0,68 ; p<0,0001)$ e a Escala de Restrição ( $r=0,60 ; p<0.0001)$ e boa validade discriminatória (known-groups) avaliada na comparação dos escores de estudantes universitárias com pacientes com transtornos alimentares'. A escala mostrou ainda diferenciar universitárias com e sem comportamento de risco para transtornos alimentares ${ }^{16}$, podendo-se pensar em estudos de rastreamento. 


\section{Desenho e participantes do estudo}

A avaliação da escala entre homens foi realizada com estudantes universitários, seguindo o modelo do desenvolvimento e validação da escala com público feminino. Para tanto, professores universitários e instituições foram contatados para possível parceria e divulgação da pesquisa. Eles foram solicitados a divulgá-la para as turmas e cursos com presença majoritária de homens.

O método de validação known-groups ${ }^{17}$ foi usado para comparar os universitários com pacientes com TA; este grupo é conhecido por problemas em relação à alimentação e, portanto, se esperava maior pontuação na escala na avaliação.

A presente pesquisa trabalhou, portanto, com dois grupos:

Grupo 1 - Estudantes universitários. Docentes de quatro instituições de ensino superior (três de São Paulo, capital, e uma de Juiz de fora, MG) foram contatados para que convidassem seus alunos do sexo masculino para participação da pesquisa e a divulgassem para colegas de outras instituições. A pesquisa foi hospedada online no site seguro SurveyMonkey ${ }^{18}$. As respostas online vieram de estudantes de $22 \mathrm{di}$ ferentes instituições (todas de São Paulo - Capital e interior); e duas das instituições primeiramente contatadas (SP e MG) participaram com respostas coletadas em sala de aula - em papel. Os alunos participantes eram de cursos diversos: estudantes de Educação Física e Biomedicina (responderam em papel), Enfermagem, Medicina, Veterinária, Biologia, Fisioterapia, Farmácia, Odontologia, Engenharia, Matemática, Rádio e TV, Ciências da Computação, Administração, Psicologia, Arquitetura, Artes Plásticas, Letras e Direito (online), constituindo, portanto, um grupo bastante eclético.

Os critérios de inclusão foram: sexo masculino, idade entre 18 e 40 anos; e de exclusão estar cursando graduação em Nutrição - porque alguns trabalhos apontam atitudes mais transtornadas entre esses estudantes ${ }^{19}$.

Os universitários responderam também a dados de caracterização como idade, peso e estatura (referidos), renda familiar e grau de instrução do chefe da sua família. Foi calculado o índice de massa corpórea (IMC) para avaliação do estado nutricional. A classificação do estado nutricional segundo IMC foi realizada de acordo com as categorias de estado nutricional da Organização Mundial da Saúde ${ }^{20}$ como baixo peso, eutrofia e sobrepeso e obesidade.

Para a análise known-groups, os universitários que pontuaram $\geq 21$ no Teste de Atitudes Alimentares (EAT-26) não foram incluídos, uma vez que esse é o ponto de corte que indica comportamento de risco para $\mathrm{TA}^{11,21}$.

Grupo 2 - Pacientes do Grupo de Atendimento a Homens com Transtornos Alimentares (GAHTA) do Programa de Transtornos Alimentares - Ambulim - do Instituto de Psiquiatria do Hospital das Clínicas da Faculdade de Medicina da Universidade de São Paulo foram convidados a preencher os instrumentos. Os critérios de inclusão foram: diagnóstico de anorexia nervosa, bulimia nervosa ou transtorno alimentar não especificado, com os critérios do Manual de Diagnósticos DSM-IV22, estabelecido por psiquiatra usando a entrevista clínica estruturada MINI23; idade entre 18 e 40 anos; e estar sem receber tratamento psiquiátrico e/ou psicológico e/ou nutricional há, pelo menos, 1 mês. Os critérios de exclusão foram apresentar quadros psicóticos; possuir abuso/ dependência de álcool e drogas; ou apresentar depressão grave. Os pacientes referiram idade e tiveram seus dados de peso e estatura aferidos pela equipe de nutrição do Ambulim para cálculo do IMC.

O cálculo amostral foi realizado no software GPower 3.1.7 utilizando um nível de significância de $p<0,05$, tamanho do efeito de $15 \%$ e poder observado de $80 \%$, totalizando a um $\mathrm{N}$ de 351 indivíduos para uma análise em modelo linear geral com dois grupos e duas covariáveis.

\section{Avaliação psicométrica e análises estatísticas}

Para analisar evidências de validade convergente, o escore na EAAT foi correlacionado com os escores de dois outros instrumentos que avaliam atitudes alimentares específicas; para tanto os universitários responderam também a EAT$26^{11,21}$ que avalia comportamentos de risco para TA, e a Escala de Restrição Alimentar - $\mathrm{RS}^{24}$ que avalia em que extensão as pessoas se preocupam com seu peso e cronicamente restringem sua alimentação de forma a controlá-lo. Supõe-se que, caso a escala seja válida para o sexo masculino, sua pontuação deva se correlacionar positivamente com os escores obtidos pelos participantes nessas outras escalas. Uma compreensão mais detalhada foi avaliada examinando as correlações de todos os fatores ou subescalas.

Estes dois instrumentos - EAT e RS - foram desenvolvidos originalmente em língua inglesa ${ }^{10,11}$, mas foram traduzidos para a língua portuguesa e mostraram boa validade discriminante e confiabilidade ${ }^{21,24}$. O estudo de Bighetti fez a validação com adolescentes do sexo feminino, mas a mesma versão foi utilizada em estudo de validação do EAT com adolescentes do sexo masculino, com adequada consistência interna $(a=0,88)^{25}$.

A análise de evidência de validade discriminatória foi realizada pelo método known-groups. Um instrumento com alta validade discriminatória é aquele que, quando administrado a amostras que sabidamente variam quanto ao constructo em questão (no caso, atitudes alimentares transtornadas), obtém pontuações significativamente diferentes para cada grupo; assim se esperava que, caso o instrumento fosse válido também para homens, os escores obtidos pelos pacientes seriam superiores àqueles obtidos por universitários sem sintomas de TA. Para tanto, os universitários que pontuaram $\geq 21$ no EAT ( $n=19 ; 8,3 \%$ ) não foram incluídos na análise.

A pontuação da EAAT total e subescalas foi também comparada entre aqueles universitários que pontuaram $<21$ 
(sem comportamento de risco para TA) e $\geq 21$ (com comportamento de risco para TA).

Para avaliar a reprodutibilidade da escala, um subgrupo foi convidado a responder novamente à EAAT aproximadamente um mês depois do primeiro preenchimento em uma análise teste-reteste; 38 universitários responderam à escala no reteste $(16,7 \%)$.

As análises foram realizadas utilizando o software SPSS 17.0 (Statistical Package for Social Science Inc., Chicago, IIIinois USA). O nível de significância adotado foi de 5\%. A normalidade das distribuições das variáveis foi testada por meio do teste de Shapiro-Wilk, e as variáveis não normais foram padronizadas por meio do Z-escore.

As pontuações da EAAT total e subescalas foram comparadas entre os universitários com e sem comportamento de risco para TA (avaliado pela EAT-26), bem como entre universitários sem risco para TA e pacientes com transtornos alimentares. Para tanto, utilizou-se um modelo linear geral (GLM), para verificar o efeito do grupo, controlando-se os resultados pela idade e IMC dos participantes. Para essas análises, apresentam-se também o tamanho do efeito (Eta Square) e o poder observado do teste.

A frequência nas categorias de estado nutricional entre os homens com e sem risco para TA foi comparada por meio do teste Qui-quadrado com correção de Yates.

A consistência interna da escala e de seus fatores foi determinada utilizando o coeficiente Alpha de Cronbach, considerando-se adequado $>0,60^{26}$.

A correlação entre a pontuação da EAAT e do EAT-26 e da RS foi avaliada por meio do coeficiente de correlação de Pearson (r). A reprodutibilidade teste-reteste da escala foi avaliada usando o coeficiente de correlação intraclasse (CCI) e o teste de Wilcoxon.

\section{CONSIDERAÇÕES ÉTICAS}

O Comitê de Ética da Faculdade de Saúde Pública da Universidade de São Paulo aprovou o protocolo de desenvolvimento, validação e aplicação da Escala de Atitudes Alimentares Transtornadas em diferentes públicos (protocolo 1351/06).

Os universitários que acessaram o site da pesquisa tiveram as informações a respeito desta na página de apresentação e deram seu consentimento assinalando "concordo" e seguindo com as respostas aos instrumentos. Aqueles que responderam em sala de aula receberam informações por escrito e assinaram o Termo de Consentimento Livre e Esclarecido para pesquisa. As instituições deram consentimento para pesquisa. Os pacientes do Ambulim foram também informados sobre a pesquisa e deram consentimento por escrito.

\section{RESULTADOS}

O grupo de universitários totalizou 228 indivíduos. Com relação ao EAT, a média de pontuação foi de 9,66 (DP 7,75), sendo que 19 universitários (8,3\%) pontuaram $\geq 21$. O perfil de idade, IMC, frequência nas categorias de estado nutricional e escores da EAAT total e subescalas - separado por universitários com e sem comportamento de risco para TA - pode ser visualizado na tabela 1. A consistência interna para escala total e seus fatores está também na tabela 1.

Tabela 1. Idade, índice de massa corpórea (IMC) e escores da Escala de Atitudes Alimentares Transtornadas (EAAT) de homens universitários $(n=228)$ - dados de tendência central e desvio-padrão, frequência nas categorias de estado nutricional

\begin{tabular}{|c|c|c|c|c|c|c|c|}
\hline & $\begin{array}{l}\text { Homens sem } \\
\text { risco para } \\
\text { transtornos } \\
\text { alimentares } \\
(\text { EAT }<21)^{*} \\
(n=209)\end{array}$ & $\begin{array}{l}\text { Homens com } \\
\text { risco para } \\
\text { transtornos } \\
\text { alimentares } \\
(E A T \geq 21)^{*} \\
(n=19)\end{array}$ & p & $\begin{array}{l}\text { Tamanho } \\
\text { efeito (Eta } \\
\text { Square) }\end{array}$ & $\begin{array}{c}\text { Poder } \\
\text { observado }\end{array}$ & Homens total & $\begin{array}{c}\text { Alpha } \\
\text { de Cronbach }\end{array}$ \\
\hline Idade & $22,15(4,01)$ & $23,16(4,03)$ & 0,298 & & & $22,24(4,01)$ & \\
\hline IMC & $22,59(7,39)$ & $22,62(10,75)$ & 0,764 & & & $22,51(7,69)$ & \\
\hline \multicolumn{8}{|l|}{ Categorias estado nutricional } \\
\hline Baixo peso (IMC $\left.<18,5 \mathrm{~kg} / \mathrm{m}^{2}\right)$ & $20(9,6 \%)$ & $3(15,8 \%)$ & $0,042^{\mathrm{a}}$ & & & $23(10,0 \%)$ & \\
\hline Eutrofia (IMC > 18,5 $\leq 24,99 \mathrm{~kg} / \mathrm{m}^{2}$ ) & $126(60,3 \%)$ & $6(31,6 \%)$ & & & & $132(57,4 \%)$ & \\
\hline Sobrepeso (IMC $\left.\geq 25 \leq 29,99 \mathrm{~kg} / \mathrm{m}^{2}\right)$ & $52(24,9 \%)$ & $6(31,6 \%)$ & & & & $58(25,2 \%)$ & \\
\hline Obesidade (IMC $\left.\geq 30 \mathrm{~kg} / \mathrm{m}^{2}\right)$ & $11(5,3 \%)$ & $4(21,1 \%)$ & & & & $15(6,5 \%)$ & \\
\hline EAAT total ${ }^{* *}$ & $55,76(10,23)$ & $78,37(14,63)$ & $<0,001$ & 0,270 & $100 \%$ & $57,64(12,36)$ & 0,63 \\
\hline EAAT Fator $1^{* *}-$ Relação com comida & $18,42(5,36)$ & $27,89(8,47)$ & $<0,001$ & 0,202 & $100 \%$ & $19.21(6,24)$ & 0,69 \\
\hline EAAT Fator $2^{* *}$ - Preocupação com comida e ganho de peso & $6,48(2,51)$ & $13,68(3,76)$ & $<0,001$ & 0,371 & $100 \%$ & $7,08(3,30)$ & 0,73 \\
\hline EAAT Fator $3^{* *}$ - Práticas restritivas e compensatórias & $4,86(1,71)$ & $7,58(3,63)$ & $<0,001$ & 0,139 & $100 \%$ & $5,09(2,07)$ & 0,27 \\
\hline EAAT Fator $4^{* *}$ - Sentimentos em relação à alimentação & $3,46(1,55)$ & $4,95(1,81)$ & 0,136 & 0,010 & $32 \%$ & $3,51(1,58)$ & 0,36 \\
\hline EAAT Fator $5^{* *}$-Conceito de alimentação normal & $22,54(5,81)$ & $25,16(6,95)$ & 0,063 & 0,015 & $46 \%$ & $22,76(5,94)$ & 0,28 \\
\hline
\end{tabular}

* Teste de Atitudes Alimentares (EAT-26); ** Quanto maior a pontuação, mais transtornadas são as atitudes.

a: $X^{2}(3)=10,05(p=0,018)-$ correção Yates $0,042$. 
A frequência encontrada nas classificações de estado nutricional entre os grupos foi comparada por meio do Qui-quadrado de Pearson, mas como duas frequências foram menores do que cinco a estatística não é precisa; a correção de Yates, no entanto, apontou associação ( $p<0,05)$.

As correlações da escala total e das subescalas com o EAT-26 e a Escala de Restrição (RS) se encontram na tabela 2.

Com relação ao Grupo 2, 28 pacientes com transtornos alimentares responderam aos instrumentos. O perfil desses pacientes e a comparação com os universitários sem risco para TA podem ser observados na tabela 3. Houve impossibilidade de obter dados de quatro pacientes quanto à idade e ao IMC; entre os demais, sete $(25,0 \%)$ tinham baixo peso; dez $(35,7 \%)$ estavam eutróficos; e sete (25,0\%), com sobrepeso. A primeira análise GLM apontou que não havia efeito da idade nem do IMC na comparação, e sim do grupo. Como houve essa impossibilidade de dados completos para todos os pacientes, os resultados da análise GLM apresentados na tabela 3 são sem covariáveis (para inclusão dos 28 pacientes com TA).

Para o reteste, houve resposta de 38 universitários e eles tinham idade e IMC similar ao grupo teste (dados não demonstrados). O escore médio no teste foi de 57,64 (DP 9,37) e, no reteste, de 54,97 (DP 12,33). O coeficiente de correlação intraclasse entre as medidas foi de $0,948(p<0,001)$, e o teste de Wilcoxon $(Z=-1,565 ; p=0,118)$.

\section{DISCUSSÃO}

O presente estudo avaliou evidências iniciais das propriedades psicométricas da Escala de Atitudes Alimentares Transtornadas para adultos do sexo masculino, concluindo que esta tem validade e reprodutibilidade aceitáveis.

A frequência de comportamento de risco de TA nesse grupo de universitários é compatível com a literatura em relação aos homens ${ }^{7}$. Observa-se que aqueles com tal comportamento de risco possuíam mais baixo peso e excesso de peso/obesidade do que os universitários sem risco - o que está de acordo com o que se sabe sobre a relação entre peso e comportamentos inadequados com relação à alimentação e ao corpo ${ }^{27,28}$.

A EAAT foi capaz de diferenciar os universitários com e sem risco para TA, considerando-se seu escore total e fatores 1, 2 e 3 - sendo que o tamanho do efeito foi acima do mínimo de 0,015 e houve grande representatividade em relação à população em geral (pelo poder observado). No entanto, a EAAT não foi diferente entre esses grupos para "sentimentos com relação à alimentação" e "conceito de alimentação normal" (fatores 4 e 5) - análise que encontrou também tamanho do efeito abaixo do mínimo e apenas médio poder observado.

Pode-se questionar o $\mathrm{N}$ amostral alcançado para o presente trabalho - abaixo dos 351 calculados previamente -

Tabela 2. Correlação entre a Escala de Atitudes Alimentares Transtornadas (EAAT) e o Teste de Atitudes Alimentares (EAT) e a Escala de Restrição (RS) na avaliação de homens universitários $(n=228)$

\begin{tabular}{lcc}
\hline EAAT & Coeficientes de correlação de Pearson $(r)$ com EAT & Coeficientes de correlação de Pearson $(r)$ com RS \\
\hline EAAT total & $0,647^{*}$ & $0,505^{*}$ \\
EAAT Fator 1 - Relação com comida & $0,509^{*}$ & $0,484^{*}$ \\
EAAT Fator 2 - Preocupação com comida e ganho de peso & $0,656^{*}$ & $0,577^{*}$ \\
EAAT Fator 3 - Práticas restritivas e compensatórias & $0,489^{*}$ & $0,367^{*}$ \\
EAAT Fator 4 - Sentimentos em relação à alimentação & $0,144^{* *}$ & 0,022 \\
EAAT Fator 5 - Conceito de alimentação normal & $0,237^{*}$ & 0,099 \\
\hline
\end{tabular}

${ }^{*} p<0,001 ;{ }^{* *} p<0,05$.

Tabela 3. Idade, índice de massa corpórea (IMC) e escores da Escala de Atitudes Alimentares Transtornadas (EAAT) de homens universitários sem risco para transtorno alimentar (TA) e pacientes com TA ( $n=28)$ - dados de tendência central e desvio-padrão

\begin{tabular}{|c|c|c|c|c|c|}
\hline & $\begin{array}{c}\text { Homens sem risco para TA } \\
- \text { EAT }<21^{*}(n=219)\end{array}$ & $\begin{array}{l}\text { Homens com TA } \\
\qquad(\mathrm{n}=28)\end{array}$ & $\mathbf{p}$ & $\begin{array}{l}\text { Tamanho efeito } \\
\text { (Eta Square) }\end{array}$ & Poder observado \\
\hline Idade & $22,15(4,01)$ & $23,09(5,81)$ & 1,000 & & $100 \%$ \\
\hline IMC & $22,59(7,39)$ & $21,80(4,11)$ & 1,000 & & $100 \%$ \\
\hline EAAT total ${ }^{* *}$ & $55,76(10,23)$ & $105,43(27,76)$ & $<0,001$ & 0,589 & $100 \%$ \\
\hline EAAT Fator $1^{* *}-$ Relação com comida & $18,42(5,36)$ & $37,54(12,37)$ & $<0,001$ & 0,472 & $100 \%$ \\
\hline EAAT Fator $2^{* *}$ - Preocupação com comida e ganho de peso & $6,48(2,51)$ & $13,89(4,60)$ & $<0,001$ & 0,419 & $100 \%$ \\
\hline EAAT Fator $3^{* *}$ - Práticas restritivas e compensatórias & $4,86(1,71)$ & $13,71(6,29)$ & $<0,001$ & 0,536 & $100 \%$ \\
\hline EAAT Fator $4^{* *}-$ Sentimentos em relação à alimentação & $3,46(1,55)$ & $8,14(4,97)$ & $<0,001$ & 0,317 & $100 \%$ \\
\hline EAAT Fator $5^{* *}$ - Conceito de alimentação normal & $22,54(5,81)$ & $32,14(10,54)$ & $<0,001$ & 0,185 & $100 \%$ \\
\hline
\end{tabular}

* Pontuação menor do que 21 no Teste de Atitudes Alimentares (EAT), ou seja, sem comportamento de risco para transtorno alimentar; ** Quanto maior a pontuação, mais transtornadas são as atitudes. 
como uma limitação para os resultados mencionados. No entanto, para validação de instrumentos, sugere-se classicamente 10 sujeitos por item do instrumento ${ }^{29}$, que foi o mínimo buscado - e alcançado - para o presente estudo. Ainda, segundo Kline ${ }^{30}, 200$ sujeitos seriam suficientes para avaliar confiabilidade de um instrumento.

Para universitárias do sexo feminino, a escala foi capaz de diferenciar aquelas com e sem risco com relação ao escore total e a todos os fatores; mas para adolescentes o fator "conceito de alimentação normal" também não diferenciou os grupos. No caso dos adolescentes, a escala também não diferenciou meninos e meninas com relação a "conceito de alimentação normal"15. Essa não diferença em relação ao fator 5 (conceito de alimentação normal) pode ser compreendida se consideradas as opiniões atuais sobre corpo e dieta. Todos de alguma forma - que parece similar entre os sexos e estágios de vida - são influenciados pelas mensagens atuais que pregam um corpo magro, "em forma e belo", e que é "construído" por uma alimentação "saudável" que prescreve a proibição de grupos e alimentos, e consumo daqueles ricos em nutrientes essenciais e pobres em gorduras e açúcares ${ }^{31}$.

Apesar de os sintomas do TA serem semelhantes em homens e mulheres, os fatores condicionantes do TA em homens parecem ser diferentes ${ }^{7}$, o que pode explicar de alguma forma o achado para os fatores 4 e 5 . De qualquer forma, na comparação de universitários sem risco com os pacientes com TA, mesmo o fator "conceito de alimentação normal" foi diferente - o mesmo aconteceu para mulheres jovens e adolescentes ${ }^{1,15}$, demonstrando que, para aqueles com diagnóstico de TA (e não risco apenas), o conceito é ainda mais rígido.

De maneira similar à escala para mulheres e adolescentes, a EAAT se correlacionou com o EAT e RS - de maneira mais fraca e sem significância nos fatores 4 e 5 para a $\mathrm{RS}^{1,15}$. As correlações oscilaram quanto aos fatores nos diferentes trabalhos de validação ${ }^{1,12-15}$, refletindo perfis diferentes entre as populações, mas com correlações moderadas para o escore total da EAT e RS atestando a convergência da EAAT e apontando que ela embarca constructos diversos dessas escalas do mesmo campo do conhecimento.

A comparação entre universitários e pacientes com TA atestou ainda que a EAAT discrimina grupos com atitudes transtornadas sabidamente diferentes - e neste caso a diferença foi válida para o escore total e os cinco fatores. E a confiabilidade teste-reteste mostrou que as respostas foram estáveis no tempo.

A consistência interna da EAAT total para essa aplicação com o sexo masculino se apresentou adequada segundo DeVellis ${ }^{26}$, mas abaixo da versão original para mulheres $(0,75)^{1}$, para adolescentes do sexo feminino e masculino 0,81 e 0,71, respectivamente ${ }^{15}$; e abaixo das versões em espanhol, inglês e japonês $(0,76 ; 0,70 ; 0,76 \text {, respectivamente })^{12-14}$. Os valores para as subescalas 3, 4 e 5 foram inadequados. A consistência desses mesmos fatores para adolescentes do sexo masculino foi também mais baixa $(0,55 ; 0,41$ e 0,38, respectivamente).

Apenas em nível de exploração, a consistência interna da EAAT foi avaliada entre os universitários excluindo-se aqueles com risco para TA, mas os valores não foram melhores; a mesma exploração dos valores apenas entre os pacientes com diagnóstico de TA encontrou valores mais adequados para todos os fatores (por exemplo, Alpha da escala total 0,80) - mas o N de 28 não é suficiente para uma exploração adequada dessa validade. De qualquer forma, tais resultados apontam para o fato de os itens da escala fazerem mais sentido para homens com TA, o que é compatível com o fato de a escala ter sido desenvolvida utilizando questões de uma população clínica com TA. Como os TA em homens são mais raros, faz sentido que homens sem TA não se reconheçam em algumas questões. Talvez exista uma diferença maior entre homens com e sem TA do que entre mulheres com e sem TA, pelas dinâmicas socioculturais de gênero que envolvem o corpo e a alimentação.

Esses achados chamam atenção para as diferenças nas atitudes alimentares transtornadas entre o público masculino e feminino. A escala foi inicialmente desenvolvida com foco no grupo feminino uma vez que os problemas alimentares são mais prevalentes em mulheres; e realmente algumas questões podem não fazer parte do "universo masculino" da mesma maneira. Scagliusi et al. ${ }^{8}$ apontam que uma das razões para não conhecermos adequadamente as questões alimentares dos homens é porque os instrumentos disponíveis são justamente focados em mulheres, não refletindo as questões masculinas. Estudos qualitativos poderiam ser conduzidos para descrever e explorar as atitudes alimentares de homens de diversos subgrupos (clínicos, não clínicos e de diversos níveis socioeconômicos, entre outros). Tais achados poderiam ser utilizados como subsídio para escalas capazes de abarcar as atitudes alimentares desse sexo.

Considerando que avaliações de TA em homens usam instrumentos muitas vezes validados apenas para mulheres, Boener et al. ${ }^{32}$ avaliaram a validade de alguns deles entre os dois sexos e afirmam que se pode presumir que as escalas tinham o mesmo sentido geral para ambos, mas as medidas foram menos confiáveis para os homens. As razões para tal achado são consistentes com o estudo atual, e não se sabe exatamente por quê. Devem-se explorar questões como: os itens são escritos com base em algum conhecimento mais feminino? Presumem experiências femininas deixando os homens sem saber exatamente como responder? São simplesmente menos interessantes para homens fazendo com que eles prestem menos atenção, resultando em respostas não atentas? ${ }^{32}$.

Embora se afirme que instrumentos para avaliação de risco e sintomatologia de TA validados para mulheres possam ser usados com homens com advertências ${ }^{32}$, acredita-se que os TA em homens são ainda mal diagnosticados e compreendidos ${ }^{9}$. Garotos podem não perceber seus compor- 
tamentos alimentares como problemáticos em função de percepções diferenciadas e também porque esses aspectos não são investigados entre eles ${ }^{28}$. Tais considerações podem ser especialmente importantes para adultos jovens, uma vez que muitos estudos focam apenas o público adolescente ${ }^{28,33}$. Cumpre ressaltar que, na população masculina, os TA desenvolvem-se mais tardiamente do que nas mulheres, sendo mais evidentes em indivíduos entre os 18 e 26 anos $^{34,35}$.

De certa maneira, os dados do presente estudo parecem apontar que homens com TA podem ter atitudes transtornadas similares às mulheres (em função de melhor consistência da escala para o grupo com TA do que sem TA, e diferenciação do grupo não clínico), mas talvez a exploração do comer transtornado e atitudes alimentares disfuncionais entre homens da população em geral peça um instrumento específico para o público masculino.

Recentemente, instrumentos com foco específico na avaliação da imagem corporal de homens foram desenvolvidos e alguns validados para o cenário brasileiro ${ }^{36-38}$, mas se desconhece instrumento específico para avaliação de atitudes alimentares de homens.

Estudos futuros nessa área devem incluir a condução de um grupo focal com homens adultos para avaliar sua opinião sobre as questões da EAAT no sentido de elas refletirem ou não suas atitudes e discutir possíveis ajustes. Tal estratégia pode melhorar a avaliação do constructo em homens. Strother et al. ${ }^{9}$ afirmam que é necessário o desenvolvimento de instrumentos de avaliação para aspectos relacionados aos TA em homens.

Este estudo tem a limitação de ter avaliado uma amostra não probabilística, que pode, portanto, ser não homogênea. De qualquer forma, as validades convergente, known-groups e confiabilidade teste-reteste da EAAT foram adequadas entre o público masculino (mesmo se considerando o grupo pequeno que respondeu ao reteste e ao $\mathrm{N}$ limitado de homens com TA - que foi o máximo possível coletado junto ao único grupo que atende esse público no Brasil). Deve-se considerar ainda a limitação metodológica relacionada aos dois diferentes formatos de aplicação do instrumento no presente trabalho: em papel em duas instituições e online nas demais, uma vez que se sabe que a resposta online gera uma resposta mais ou menos fidedigna. Para escala original em português e validação em espanhol e japonês, as respostas foram em papel; por outro lado, a validação em inglês e para adolescentes foi realizada com respostas online - por questões de acesso ao público. Dessa forma, acredita-se que a escala possa ser aplicada em ambos os formatos - embora a diferença entre esses métodos de coleta deva ser posteriormente mais bem avaliada.

Como a EAAT é de fácil aplicação, pode completar estudos sobre atitudes alimentares entre homens com TA e ampliar a compreensão do constructo entre homens de população não clínica, considerando-se que não há instru- mento específico para esse público e as limitações daqueles avaliados - como o EAT ${ }^{1}$.

Recomendam-se o ajuste da EAAT após discussão com grupo de homens e o desenvolvimento de outros instrumentos específicos para o gênero masculino, buscando cobrir as lacunas de conhecimento existentes a respeito do comer transtornado e da relação para com o alimento desse público.

\section{CONTRIBUIÇÕES INDIVIDUAIS}

Marle dos Santos Alvarenga - Foi responsável pela concepção do estudo, desenho, análise dos dados, escrita e revisão final depois das contribuições dos coautores.

Pedro H. B. Carvalho - Colaborou com a coleta e organização do banco de dados, interpretação dos resultados e revisão crítica do conteúdo.

Sonia T. Philippi - Foi supervisora do trabalho original de desenvolvimento e validação da escala e participou da interpretação dos resultados e revisão crítica do conteúdo.

Fernanda B. Scagliusi - Foi coautora do trabalho original de desenvolvimento e validação da escala e contribuiu para a concepção do estudo, interpretação dos resultados e revisão crítica e intelectual de seu conteúdo.

Todos aprovaram a versão final do manuscrito.

\section{CONFLITOS DE INTERESSE}

Os autores declaram não haver conflito de interesses.

\section{AGRADECIMENTOS}

Os autores agradecem a Fundação para Pesquisa do Estado de São Paulo - Fapesp (Processo 06/56850-9), pelo apoio concedido ao primeiro autor deste trabalho e que deu suporte à pesquisa; agradecem também às Instituições participantes pela parceria.

\section{FINANCIAMENTO}

Fundação para Pesquisa do Estado de São Paulo (Fapesp) (Processo 06/56850-9); Conselho Nacional de Desenvolvimento Científico e Tecnológico (CNPq) (no 309121/2012-4).

\section{REFERÊNCIAS}

1. Alvarenga MS, Scagliusi FB, Philippi ST. Development and validity of the disordered eating attitude scale. Percept Mot Skills. 2010;110(2):379-95. 
2. Sunday SR, Einhorn A, Halmi KA. Relationship of perceived macronutrient and caloric content to affective cognitions about food in eating-disordered, restrained, and unrestrained subjects. Am J Clin Nutr. 1992;55:362-71.

3. American Dietetic Association. Position of the American Dietetic Association: nutrition intervention in the treatment of eating disorders. J Am Diet Assoc. 2011;111:1236-41.

4. Germov J, Williams $L$. The epidemic of dieting women: the need for a sociological approach to food and nutrition. Appetite. 1996;27:97-108.

5. Rozin P, Bauer R, Catanese D. Food and life, pleasure and worry, among American college students: gender differences and regional similarities. J Pers Soc Psychol. 2003;85(1):132-41.

6. Fabbri AD, Sato PM, Queiroz GKO, Silva MB. Transtornos alimentares em homens: quais são as diferenças em relação às mulheres? In: Alvarenga MS, Philippi ST. Nutrição e transtornos alimentares - Avaliação e tratamento. São Paulo: Manole; 2010.

7. Jones WR, Morgan JF. Eating disorders in men: a review of the literature. J Public Mental Health. 2010;9(2):23-31.

8. Scagliusi FB, Nakagawa KA, Campos RM, Kotait M, Fabbri A, Sato P, et al. Nutritional knowledge, eating attitudes and chronic dietary restraint among men with eating disorders. Appetite. 2009;53:446-9.

9. Strother E, Lemberg R, Stanford SC, Turberville D. Eating disorders in men: underdiagnosed, undertreated, and misunderstood. Eat Disord. 2012;20:346-55.

10. Herman (P, Mack D. Restrained and unrestrained eating. J Pers. 1975;43(4):647-60.

11. Garner DM, Olmested MP, Bohr Y, Garfinkel PE. The Eating Attitude Test: psychometric features and clinical correlates. Psychol Med. 1982;12:871-79.

12. Alvarenga MS, Pereira RF, Scagliusi FB, Philippi ST, Estima CC, Croll J. Psychometric evaluation of the Disordered Eating Attitude Scale (DEAS) - English version. Appetite. 2010:55:374-6

13. Alvarenga MS, Francischi R, Fontes F, Scagliusi FB, Philippi ST. Adaptación y validación al Espanol del Disordered Eating Attitude Scale (DEAS). Perspect Nutr Hum. 2010;12(1):11-23.

14. Chisuwa N, Shimai S, Haruki T, Alvarenga MS. Development of the Japanese Version of the Disordered Eating Attitude Scale (DEAS): Validity and reliability among female university students. School Health. 2013;9:14-22.

15. Alvarenga MS, Koritar P, Vanessa P, Figueiredo M, Fleitlich-Bilyk B, Philippi ST, et al. Disordered eating in Brazilian adolescents: findings from the psychometric evaluation of the Disordered Eating Attitude Scale. Appetite. Submetido.

16. Alvarenga MS, Scagliusi FB, Philippi ST. Eating attitudes of female Brazilian university students with eating disorder risk behavior. J Behav Health Social Issues. 2010;2(1):47-54.

17. Hattie J, Cooksey RW. Procedures for assessing the validities of tests using the "knowngroups" method. Appl Psychol Meas. 1984;8:295-305.

18. Survey Monkey (2011). Free online survey \& questionnaire tool. Disponível em: <www. surveymonkey.com>. Acesso em: 10 julho, 2011

19. Alvarenga MS, Scagliusi FB, Philippi ST. Comparison of eating attitudes among university students from the five Brazilian regions. Ciên Saúde Coletiva. 2011;17(2):435-44.

20. World Health Organization (WHO). Global database on Body Mass Index. Geneva: WHO; 2006. Disponível em: http://apps.who.int/bmi/index.jsp?introPage=intro_3.html. Acesso em: 30 junho, 2013.
21. Bighetti F, Santos CB, Santos JE, Ribeiro RPP. Tradução e validação do Eating Attitudes Test em adolescentes do sexo feminino de Ribeirão Preto, SP. J Bras Psiquiatr. 2004;53:339-46.

22. American Psychiatric Association (APA). Diagnostic and Statistical Manual of Mental Disorders. 4th ed. Washington DC; 1994.

23. Amorim P. Mini International Neuropsychiatric Interview (MINI): validação de entrevista breve para diagnóstico de transtornos mentais. Rev Bras Psiquiatr. 2000;22:106-15.

24. Scagliusi FB, Polacow VO, Cordás TA, Coelho D, Alvarenga MS, Philippi ST, et al. Test-retest reliability and discriminant validity of the Restraint Scale translated into Portuguese. Eat Behav. 2005;6:85-93

25. Fortes LS, Amaral ACS, Almeida SS, Conti MA, Ferreira MEC. Qualidades psicométricas do Eating Attitudes Test (EAT-26) para adolescentes brasileiros do sexo masculino. Psic Teor Pesq. Submetido.

26. DeVellis RF. Scale development: theory and applications. Newbury Park, CA: SAGE Publications; 1991

27. Alvarenga MS, Lourenço BH, Philippi ST, Scagliusi FB. Disordered eating among Brazilian female college students. Cad Saúde Pública. 2013;29(5):879-88.

28. Dominé F, Berchtold A, AkréC, Pierre-André M, Suris JC. Disordered eating behaviors: What about boys? J Adoles Health. 2009:44:111-7.

29. Kerlinger FN. Foundations of behavioral research. New York: Holt, Rinehard and Winston; 1986.

30. Kline P. A handbook of test construction. Australia: Law Book Co of Australasia; 1986.

31. Paquette MC. Perceptions of healthy eating: state of knowledge and research gaps. Can J Public Health. 2005; 96(Suppl 3):S15-9, S16-21.

32. Boener LM, Spillane NS, Anderson KG, Smith GT. Similarities and differences between women and men on eating disorder risk factors and symptom measures. Eat Behaviors. 2004;5(3):209-22.

33. Muise AM, Stein DG, Arbess G. Eating disorders in adolescent boys: a review of the adolescent and young adult literature. J Adoles Health. 2003;33(6):427-35.

34. Braun DL, Sunday SR, Huang A, Halmi KA. More male seek treatment for eating disorders. Int J Eat Disord. 1999;25(4):415-24.

35. Carlat D, Camargo C, Herzog DB. Eating disorders in males: report on 135 patients. Am J Psychiatr. 1997;154(8):831-43.

36. Campana ANNB, Tavares MCGCF, Swami V, Silva D. An examination of the psychometric properties of Brazilian Portuguese Translations of the Drive for Muscularity Scale, the Swansea Muscularity Attitudes Questionnaire, and the Masculine Body Ideal Distress Scale. Psychol Men Masculinity. 2012;3: no pagination specified.

37. Carvalho PHB, Ferreira MEC, Kotait M, Teixeira PC, Hearst N, Cordás TA, et al. Equivalências conceitual, semântica e instrumental: análises preliminares da versão em português (Brasil) da Male Body Dissatisfaction Scale (MBDS). Cad Saúde Pública. 2013:29:403-9.

38. Carvalho PHB, Conti MA, Cordás TA, Ferreira MEC. Tradução para o português (Brasil), equivalência semântica e consistência interna do Male Body Checking Questionnaire (MBCQ). Rev Psiquiatr Clin. 2012;39:74-5. 